\title{
The predictive value of microRNA-126 in relation to first line treatment with capecitabine and oxaliplatin in patients with metastatic colorectal cancer
}

Torben Frøstrup Hansen ${ }^{1,3^{*}}$, Flemming Brandt Sørensen ${ }^{2}$, Jan Lindebjerg ${ }^{2}$ and Anders Jakobsen ${ }^{1}$

\begin{abstract}
Background: MicroRNA-126 is the only microRNA (miRNA) known to be endothelial cell-specific influencing angiogenesis in several ways. The aim of the present study was to analyse the possible predictive value of miRNA-126 in relation to first line capecitabine and oxaliplatin (XELOX) in patients with metastatic colorectal cancer (mCRC).

Methods: The study included 89 patients with mCRC. In situ hybridization (ISH) was performed to detect miRNA-126 in formalin-fixed paraffin embedded tissue from primary tumours. The expression of miRNA-126, area per image $\left(\mu \mathrm{m}^{2}\right)$, was measured using image analysis. Clinical response was evaluated according to RECIST. Progression free survival (PFS) was compared using the Kaplan-Meier method and the log rank test. Tumours were classified as low or high miRNA-126 expressing tumours using the median value from the patients with response as cut-off.
\end{abstract}

Results: The median miRNA-126 expression level was significantly higher in patients responding to XELOX, 3629 $\mu \mathrm{m}^{2}$ (95\% Cl, 2566-4846), compared to the patients not responding, $1670 \mu \mathrm{m}^{2}$ (95\% Cl, 1436-2041), $p<0.0001$. The positive predictive value was $90 \%$, and the negative predictive value was $71 \%$. The median PFS of patients with high expressing tumours was 11.5 months ( $95 \%$ Cl, 9.0-12.7 months) compared to 6.0 months (95\% Cl, 4.8-6.9 months) for patients with low expressing tumours, $p<0.0001$.

Conclusions: Angiogenesis quantified by ISH of miRNA-126 was related to response to first line XELOX in patients with $\mathrm{mCRC}$, translating to a significant difference in PFS. The predictive value of miRNA-126 remains to be further elucidated in prospective studies.

Keywords: Angiogenesis, Chemotherapy, Colorectal neoplasms, microRNAs, Predictive biomarkers

\section{Background}

In the recent years, a rapidly growing number of treatment modalities have become available for the treatment of patients with metastatic colorectal cancer (mCRC). It is therefore more important than ever to identify the right patients for the right treatment. Combination chemotherapy constitutes the backbone of mCRC treatment and combined with anti-angiogenetic therapy this is a common first line choice.

\footnotetext{
* Correspondence: torben.hansen@slb.regionsyddanmark.dk

'Department of Oncology, Vejle Hospital, Kabbeltoft 25, 7100 Vejle, Denmark Full list of author information is available at the end of the article
}

In the search for potential biomarkers, angiogenesis, the formation of new blood vessels from pre-existing vessels, has been addressed for obvious reasons, including the introduction of anti-angiogenetic treatment. Furthermore, the important role of angiogenesis in the growth and dissemination of malignant tumours [1-3] argues for testing biomarkers related to the neoformation of the vasculature in malignancies.

MicroRNAs (miRNAs) are a group of small, noncoding RNAs that regulate several biological functions, and increasing evidence supports a pivotal role in the regulation of pathological processes as well $[4,5]$. The 
number of reports on miRNAs and their involvement in CRC pathogenesis is rapidly growing [6] and a relationship between miRNA expression patterns and response to cytotoxic treatment has also been demonstrated [7-10]. MicroRNA-126 [GenBank: NR_029695] is so far the only identified endothelial cell (EC)-specific miRNA [11] and its important role in regulating angiogenesis has been demonstrated in a few studies [12-14]. High levels of miRNA-126 expression have been correlated with increased vascular endothelial growth factor A (VEGF-A) mediated signalling in ECs and a higher blood vessel integrity $[12,14,15]$. Thus, current evidence seems to support a close relationship between miRNA-126 and physiological angiogenesis, but the mechanism of the regulation under different normal and malignant conditions remains to be elucidated. MicroRNA-126 is often referred to as a tumour suppressor in primary tumours and cancer cell lines [16-20]. Thus, inhibition of cancer cell-growth has been demonstrated after restoration of miRNA-126 levels $[17,19,21]$. Based on the current knowledge about angiogenesis, blood vessel structure, interstitial tumour pressure and their influence on the delivery of chemotherapy to the tumour cells [22-25], one could hypothesise that miRNA-126 would likewise harbour predictive information.

The aim of this study was to analyse the possible predictive value of miRNA-126 in patients with mCRC in relation to first line treatment with capecitabine and oxaliplatin (XELOX).

\section{Methods}

Study population, sampling and treatment regimen

The study included 89 patients with mCRC histologically verified at the Department of Clinical Pathology, Vejle Hospital, Denmark (Table 1). First line chemotherapy was initiated in the period from May 2004 to December 2009. Pre-treatment examination consisted of standard hematologic parameters and a CT scan of the chest and abdomen or a chest $\mathrm{x}$-ray and ultrasound of the abdomen. All patients received a minimum of 3 cycles of XELOX. Formalin fixed paraffin embedded (FFPE) tumour tissue originating from the primary tumour was available from all patients. Patients having received preoperative chemoradiation of rectal cancer were not included. All patients meeting these criteria were offered inclusion in the study.

Two patients had a previous diagnosis of breast cancer, but had no sign of relapse at the time of diagnosis of their CRC, and their survival data were consequently left uncensored. No patients were diagnosed with a new malignancy after their CRC diagnosis. Progression free survival data were censored in 10 cases. Six patients initiated a second-line treatment before progression and
Table 1 Patient characteristics at time of diagnosis

\begin{tabular}{|c|c|c|c|}
\hline & \multirow{2}{*}{$\begin{array}{l}\text { Number (\%) } \\
\qquad(n=89)\end{array}$} & \multicolumn{2}{|c|}{$\begin{array}{c}\text { miRNA-126 expression level } \\
\left(\mu \mathrm{m}^{2}\right)\end{array}$} \\
\hline & & Median $(95 \% \mathrm{Cl})$ & $p$ - value \\
\hline \multicolumn{4}{|l|}{ Sex } \\
\hline Male & $45(51)$ & $2533(1690-3285)$ & 0.84 \\
\hline Female & $44(49)$ & $2354(1650-3163)$ & \\
\hline \multicolumn{4}{|l|}{ Age (years) } \\
\hline Mean (SD) & $63.2(8.2)$ & & \\
\hline Range & $24-80$ & & \\
\hline$>$ mean & $49(55)$ & $2088(1556-3223)$ & 0.56 \\
\hline$<$ mean & $40(45)$ & $2531(1896-3355)$ & \\
\hline \multicolumn{4}{|c|}{$\overline{\text { ECOG Performance status }}$} \\
\hline 0 & $37(42)$ & $2041(1551-3163)$ & 0.40 \\
\hline $1-2$ & $52(58)$ & $2535(1708-3489)$ & \\
\hline \multicolumn{4}{|l|}{ Localization } \\
\hline Rectum & $18(20)$ & $3038(1690-4030)$ & 0.37 \\
\hline Colon & $71(80)$ & $2141(1650-2638)$ & \\
\hline Left colon & $47(66)$ & $2141(1589-2818)$ & 0.99 \\
\hline Right colon & $24(34)$ & $2232(1452-3355)$ & \\
\hline \multicolumn{4}{|l|}{ MSI status* } \\
\hline MSI & $8(11)$ & $2193(1374-5421)$ & 0.61 \\
\hline MSS & $62(89)$ & $2355(1705-2818)$ & \\
\hline \multicolumn{4}{|l|}{ Metastatic sites* } \\
\hline 1 & $27(31)$ & $3575(3163-4521)$ & $<0.0001$ \\
\hline$\geq 2$ & $59(69)$ & $1857(151-2376)$ & \\
\hline
\end{tabular}

Cl confidence interval, SD standard deviation, ECOG Eastern Cooperative Oncology Group, MSI microsatellite instability, MSS, microsatellite stable ${ }^{*}$ MSI status was not assessed in all patients

**A reliable estimate of the number of metastatic sites was not possible in 3 patients

data were censored from the first day of their new treatment. Two patients underwent radio frequency ablation, and two patients had surgical resection of their liver metastases and their data were censored from the day of the intervention.

Immediately after surgery the removed bowel segment was brought to the Department of Clinical Pathology and a pathologist sampled tissue from the tumour. Samples intended for later in situ hybridization (ISH) analyses followed routine fixation and paraffin embedding. Postoperatively, the tumours were histologically classified and staged according to the pTNM system. Information regarding patient characteristics, relapse status and survival were based on patient records, pathology reports, and a national registry based on central person registration numbers.

All patients received the same treatment (XELOX), which consisted of a 2-hour intravenous infusion of oxaliplatin $130 \mathrm{mg} / \mathrm{m}^{2}$ on day 1 followed by oral capecitabine $1000 \mathrm{mg} / \mathrm{m}^{2}$ twice daily on days 1 through 14 (28 
doses) of a 21-days cycle. Treatment was continued until disease progression or unacceptable toxicity.

The study was approved by the Regional Scientific Ethical Committee for Southern Denmark according to Danish law, J.nr. S-VF-20040047, and informed consent was obtained from all patients enrolled in the study.

\section{Evaluation and tumour response criteria}

Treatment response, according to RECIST (version 1.0), was assessed every 9 weeks with clinical and radiologic examination using $\mathrm{CT}$ scan of the chest and abdomen (86 patients) or a chest $\mathrm{x}$-ray and ultrasound of the abdomen (3 patients). Responding patients were classified as having either complete response (CR) or partial response (PR). Patients with stable disease (SD) or progressive disease (PD) were defined as non-responders. All CT scans were evaluated by the same investigator (TFH).

\section{LNA probe, in situ hybridization, image analysis and quantification}

The entire ISH procedure and the subsequent quantification was performed at Exiqon, Vedbaek, Denmark and followed previously described procedures [26,27].

In brief, the ISH analysis was carried out on $6-\mu \mathrm{m}$ thick FFPE tissue sections containing CRC tissue, using a double digoxigenin (DIG)-labelled mercury Locked Nucleic Acid (LNA) probe (LNA ${ }^{\mathrm{TM}}$ microRNA detection probe, Exiqon, Vedbaek, Denmark) specific for human miRNA-126. Tissue sectioning was performed in an RNase-free environment.

An initial assay optimization was performed on nine sections from five CRC samples in order to determine the optimal probe concentration and hybridization temperature. The ISH assay involved a protein-K treatment $(15 \mu \mathrm{g} / \mathrm{ml})$ followed by a hybridization step, using the LNA probe at $57^{\circ} \mathrm{C}$ and stringent washes. The DIGlabelled probe was detected with alkaline phosphatase conjugated sheep anti-DIG Fab fragments followed by NBT-BCIP chromogenic staining and nuclear fast red staining.

For image acquisition up to 25 random, systematically positioned images were collected from the tumour slide. Initially, the whole tumour area was encircled to avoid ulcerating, necrotic and normal tissue. Images containing distorted tissue and staining artefacts were deleted and only images with cancer cells and tumour stroma were included. A corresponding pixel classifier included the following colours for the identification of the stained histological structures. Intense blue (the ISH signal), weak blue (background ISH signal), red (nuclear stain), red spot (mucin), black spot (artefact), unstained (no-tissue and erythrocytes). The area of the ISH signal $\left(\mu \mathrm{m}^{2}\right.$ per image), was used as the parameter reflecting
miRNA-126 expression levels, and the mean score from the sampled images (at $\times 20$ magnification) was used for each patient. Figure 1 provides examples of the underlying pixel classifier. The entire ISH procedure was performed by a staff unaware of the clinical parameters.

\section{Statistical analysis}

Fisher's exact test was used for two-group comparisons. Median values were compared using the Wilcoxon rank sum test. Progression free survival (PFS) was defined as the time from start of treatment until the first documented tumour progression or death. Overall survival (OS) was defined as the time from start of treatment until death. All survival data were complete. Survival curves were illustrated according to the Kaplan-Meier method and the logrank test was used to test for differences between the groups. All statistical calculations were carried out using the NCSS statistical software (NCSS Statistical Software, Kaysville, UT 84037, USA, version 2007). $P$ values $<0.05$ were considered significant, and all tests were two-sided.

\section{Results}

The miRNA-126 ISH resulted in a strong signal in various vessels throughout the tumour in all the samples (Figure 1). No apparent unspecific signals were detected from the extracellular matrix, but background signal was detected in a few samples caused by tumour sections being too thick.

\section{Patient characteristics}

The patient characteristics along with the miRNA-126 expression levels in the primary tumours are shown in Table 1. A significant relationship was demonstrated between the number of metastatic sites and the median miRNA-126 expression level. The presence of two or more metastatic sites was related to lower miRNA-126 expression levels compared to the expression levels in patients with metastases limited to one site.

\section{The predictive value of miRNA-126 in relation to XELOX}

Eighty-three patients were included in the analyses of the predictive value of miRNA-126 in relation to first line XELOX in mCRC. Five patients were excluded due to the fact that XELOX was given as second or third line treatment, and one patient was excluded because of insufficient response evaluation according to the RECIST criteria.

The distribution of miRNA-126 expression levels according to response is shown in Figure 2. The median miRNA expression level was significantly higher in the responding patients, 3629 (95\% CI 2566-4846) compared to the non-responding patients, 1670 (95\% CI 1436-2041), $p=5 \times 10^{-6}$. The largest difference in 

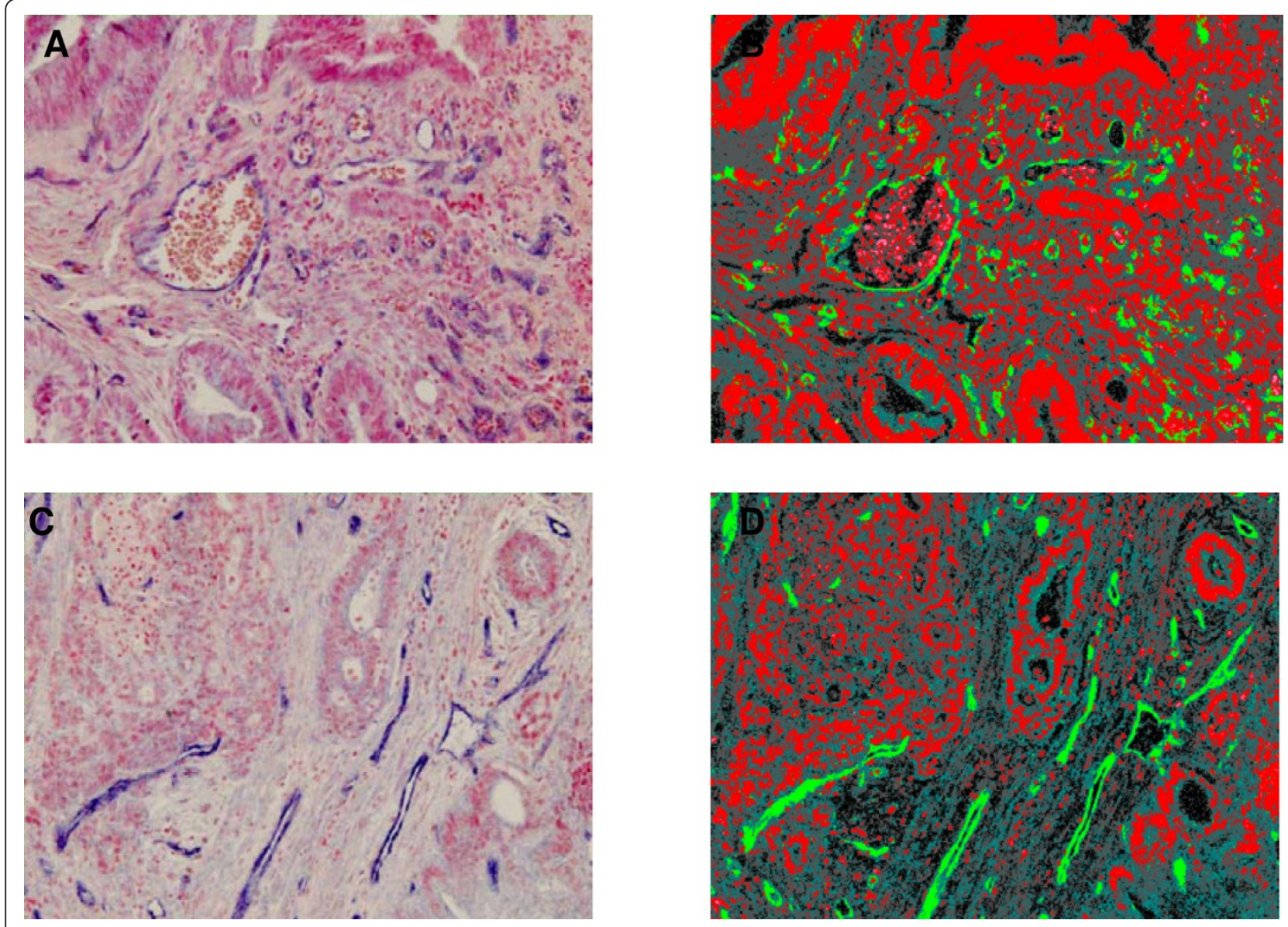

Figure 1 In situ hybridization (ISH) visualising the expression of miRNA-126 in tumour vasculature. A represents an image from a patient with colon cancer in which the mean ISH signal (the blue signal) from all the analysed images resulted in an area of $4280 \mu \mathrm{m}^{2}$. B the pixel classifier corresponding to image A. C also represents an image from a patient with colon cancer with a mean ISH signal of $2736 \mu \mathrm{m}^{2}$. D the pixel classifier corresponding to image $C$.
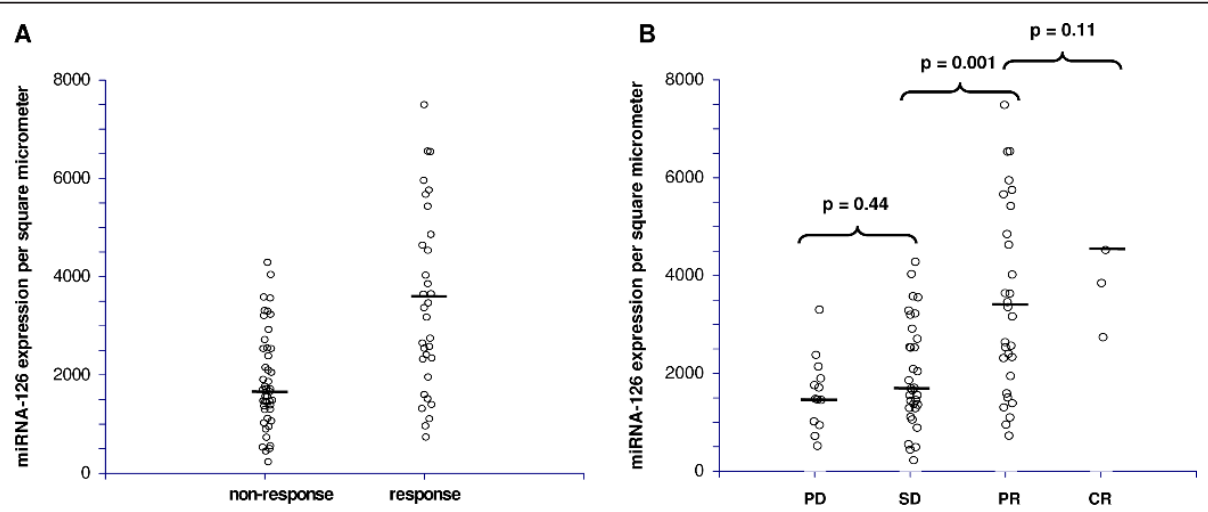

Figure 2 Relationship between miRNA-126 and response. (A) Distribution of miRNA-126 expression levels according to response or nonresponse to first line XELOX in patients with $m C R C$, black bars represent the medians, $n=83$. The difference between the medians in the two groups was significant, $p=0.000005$. (B) Illustration of the same distribution divided into the four response groups (PD; progressive disease, SD; stable disease, PR; partial response and CR; complete response). A significant difference between the median miRNA-126 expression levels in the patients achieving SD compared to PR was observed, $p=0.001$. The miRNA-126 expression levels from five patients (outliers, levels ranging from 16775-40284) are not shown in the figures for graphical reasons only, but were included in the statistics. Two of these patients achieved PR and the remaining three $C R$. 
median miRNA-126 expression levels was demonstrated when comparing patients achieving SD to patients achieving PR.

In order to assess the predictive value of the miRNA$126 \mathrm{ISH}$ analysis in the context of predicting response to first line XELOX treatment, patients were divided into two groups. The median value $\left(3629 \mu \mathrm{m}^{2}\right)$ from the response group was used as cut-off. Applying this strategy, the predictive value of a positive test $\left(>3629 \mu \mathrm{m}^{2}\right)$ was $19 / 21=90 \%$, and of a negative test $\left(<3629 \mu \mathrm{m}^{2}\right)$ was $44 / 62=71 \%$.

The same division of the patients was used in the subsequent PFS analysis (Figure 3A). The difference in response rates between the two groups reflected a significant difference in PFS, $p<0.0001$. The median PFS for patients with high miRNA-126 expression levels was 11.5 months (95\% CI 9.0-12.7 months) compared to 6.0 months (95\% CI 4.8-6.9 months) for patients with low expression levels. The difference in PFS translated into a significant difference in OS as well, $p=0.0021$ (Figure $3 \mathrm{~B})$. The median OS in the group with high miRNA-126 expression was 26.2 months (95\% CI 21.8-32.8 months) compared to 16.8 months (95\% CI 13.8-19.1 months) in the group with low miRNA-126 expression.

The median overall survival for the entire cohort was 18.5 months (95\% CI 16.7-21.8 months).

A subgroup analysis comparing patients with colon cancer and rectum cancer did not change any of the presented results.

\section{Discussion}

An increasing number of pre-clinical studies have demonstrated a pivotal role of miRA-126 in regulating angiogenesis, a process that has also been related to the delivery and the efficacy of chemotherapy. Little is known, though, about the in vivo localisation of miRNA-126 in human CRC tissue samples, but the present results support the previous studies indicating specificity towards ECs. In the present study we analysed the possible predictive value of miRNA-126 in relation to first line XELOX therapy in patients with mCRC, using a quantitative analysis of the miRNA-126 expression level based on ISH analyses of tumour sections from the primary tumour. This method has recently demonstrated its reliability in predicting short disease-free survival in stage II colon cancer patients [26].

A significant relationship between miRNA-126 expression levels and number of metastatic sites was demonstrated. The miRNA-126 expression level was significantly lower in patients with two or more metastatic sites compared to the patients with disease limited to only one metastatic location. This could indicate that the molecular genetic features of the tumour cells originating from the primary tumours with low levels of miRNA-126 are related to the ease by which these circulating tumour cells invade tissues and gives rise to distant metastases. This seems to apply rather well with the conception of miRNA-126 functioning as a tumour suppressor.

In the present study a significant relationship between miRNA-126 expression levels in the primary tumour and response to first line XELOX treatment was demonstrated for patients with mCRC. Previous studies have also reported on predictive value of markers related to angiogenesis in patients with $\mathrm{mCRC}[28,29]$. The present marker, miRNA-126, is related to vessel integrity [15], and correlations between vessel structure and response to chemotherapy have previously been demonstrated $[24,25]$ supporting the plausibility of the present results. Furthermore, a study by Zhou et al. [9] reported on changes of miRNA expression profiles in colon cancer cell lines following exposure to XELOX. Low expression levels of miRNA-126 may therefore represent
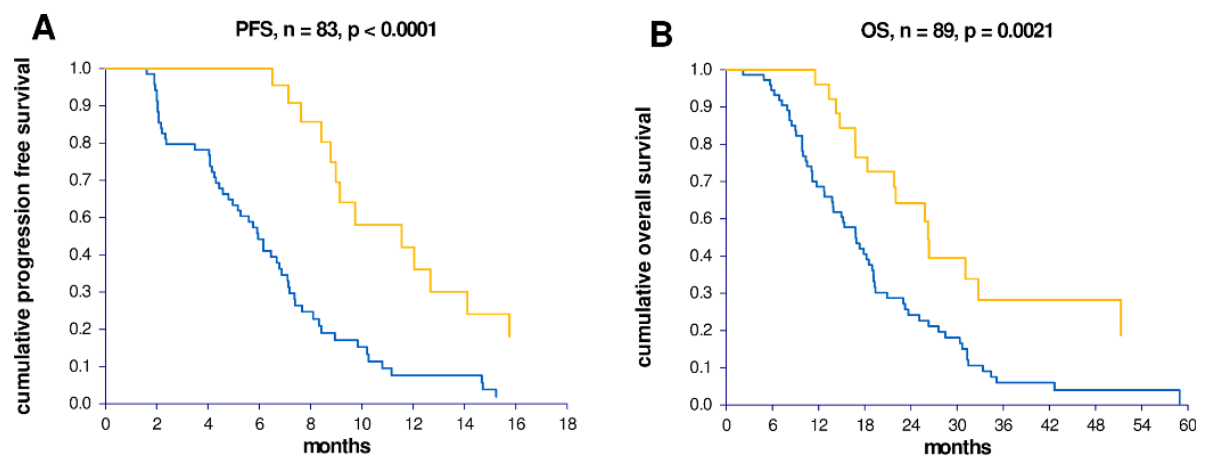

Figure 3 Kaplan-Meier survival curves according to miRNA-126 expression levels. (A) Illustrates the progression free survival (PFS) curves and (B) the overall survival (OS) curves. The yellow lines represent patients with high miRNA-126 expression levels and the blue lines patients with low miRNA-126 expression. The difference between the curves was significant in the PFS comparison, $p<0.0001$, as well as in the OS comparison, $p=0.0021$. 
tumour vessels with lower integrity and the expected increase in the interstitial tumour pressure that follows may explain the lower response rates seen in these patients.

Dividing the patients by cut-off method, i.e. median miRNA-126 expression level, demonstrated that the observed relationship with response rates was also translated into a corresponding difference in PFS. A difference in OS was also demonstrated, when comparing patients with low and high miRNA-126 expression levels. The relationship with OS may indicate that miRNA-126 has a prognostic importance in addition to the predictive value. We did not find it indicated to perform a multivariate survival analysis adjusting for parameters of prognostic importance in stage I through III disease in this cohort of patients with stage IV disease. In the study by Díaz et al. no prognostic value of miRNA-126 was demonstrated in patients with stage I to III colon cancer [30]. The disease stage of the included patients constitutes one of the major differences between their study and ours, which included patients with stage IV disease only. Schepeler et al. showed that miRNA-126 was up-regulated in patients with subsequent recurrence compared to patients with no recurrence in a cohort of 37 patients operated for stage II colon cancer with microsatellite stable tumours only [31]. Differences in methods, sample size, and stage of the disease again represent the most obvious explanations of these discrepancies, but the results from the three studies indicate that the possible prognostic value of miRNA-126 remains to be further clarified. If indeed, assessment of miRNA-126 expression in the primary tumour from patients with CRC harbours prognostic value, one would expect a better prognosis for patients with higher expression levels of this miRNA if functioning as a tumour suppressor.

The present study has the standard limitations of a retrospective study; a rather limited sample size and results obtained form a single institution. Having said that it also represents a well argued hypothesis, a reliable method, and results of clinical relevance in line with the preclinical literature.

An alternative technique to quantify angiogenesis is presented based on ISH of miRNA-126 in primary tumour tissue and using a specific LNA based probe and new quantitative systematic image analyses. This approach resulted in an estimate related to disease characteristics, the response to chemotherapy, and the prognosis of patients with mCRC. The new application of the ISH method presented here indicated that miRNA126 may be an important predictive marker to chemotherapy applied in the clinical setting, but the results call for validation in a prospective trial. Furthermore, it shall be interesting to analyse the possible predictive value of miRNA-126 in patients with mCRC treated with chemotherapy combined with anti-VEGF-A.

\section{Abbreviations}

mCRC: metastatic colorectal cancer; miRNA: microRNA; CRC: colorectal cancer; EC: endothelial cell; VEGF-A: vascular endothelial growth factor A; XELOX: capecitabine and oxaliplatin; CT: computer tomografi; FFPE: formalin fixed paraffin embedded; ISH: in situ hybridization; RECIST: response evaluation criteria in solid tumors; CR: complete response; PR: partial response; SD: stable disease; PD: progressive disease; DIG: double digoxigenin; LNA: locked nucleic acid; PFS: progression free survival; OS: overall survival; $\mathrm{Cl}$ : confidence interval.

\section{Acknowledgements}

We are very thankful for the technical assistance provided by, Birgit Roed Sørensen and Stine Jørgensen and for the writing assistance provided by Karin Larsen. Furthermore, we thank Boye Schnack Nielsen (Exiqon A/S, Denmark) for optimising the miRNA in situ hybridization method and for assisting in the interpretation of the results.

This study was supported by The Cancer Foundation and The Danish Council for Independent Research, which had no influence on any part of the study.

\section{Author details}

${ }^{1}$ Department of Oncology, Vejle Hospital, Kabbeltoft 25, 7100 Vejle, Denmark. 2Department of Clinical Pathology, Vejle Hospital, Kabbeltoft 25, 7100 Vejle, Denmark. ${ }^{3}$ Danish Colorectal Cancer Group South, Vejle Hospital, Kabbeltoft 25, 7100 Vejle, Denmark.

\section{Authors' contributions}

All authors contributed equally to the conception and design. TFH performed the data evaluation, interpretation and manuscript drafting. FBS, $\mathrm{J}$ and $\mathrm{AJ}$ performed critical revisions. All authors read and approved the final manuscript.

\section{Competing interests}

The authors declare that they have no competing interests.

Received: 17 August 2011 Accepted: 8 March 2012

Published: 8 March 2012

\section{References}

1. Folkman $\mathrm{J}$ : What is the evidence that tumors are angiogenesis dependent? J Natl Cancer Inst 1990, 82:4-6.

2. Carmeliet P: Angiogenesis in health and disease. Nat Med 2003, 9:653-660.

3. Folkman J: Tumor angiogenesis: therapeutic implications. N Engl J Med 1971, 285:1182-1186.

4. Filipowicz W, Bhattacharyya SN, Sonenberg N: Mechanisms of posttranscriptional regulation by microRNAs: are the answers in sight? Nat Rev Genet 2008, 9:102-114.

5. Esquela-Kerscher A, Slack FJ: Oncomirs - microRNAs with a role in cancer. Nat Rev Cancer 2006, 6:259-269.

6. Slaby O, Svoboda M, Michalek J, Vyzula R: MicroRNAs in colorectal cancer: translation of molecular biology into clinical application. Mol Cancer 2009, 8:102.

7. Rossi L, Bonmassar E, Faraoni I: Modification of miR gene expression pattern in human colon cancer cells following exposure to 5-fluorouracil in vitro. Pharmacol Res 2007, 56:248-253.

8. Svoboda M, Izakovicova HL, Sefr R, Vrtkova I, Kocakova I, Tichy B, Dvorak J: Micro-RNAs miR125b and miR137 are frequently upregulated in response to capecitabine chemoradiotherapy of rectal cancer. Int J Oncol 2008, 33:541-547.

9. Zhou J, Zhou Y, Yin B, Hao W, Zhao L, Ju W, Bai C: 5-Fluorouracil and oxaliplatin modify the expression profiles of microRNAs in human colon cancer cells in vitro. Oncol Rep 2010, 23:121-128.

10. Nakajima G, Hayashi K, Xi Y, Kudo K, Uchida K, Takasaki K, Yamamoto M, Ju J: Non-coding MicroRNAs hsa-let-7 $g$ and hsa-miR-181b are Associated with Chemoresponse to S-1 in Colon Cancer. Cancer Genomics Proteomics 2006, 3:317-324. 
11. Wienholds E, Kloosterman WP, Miska E, varez-Saavedra E, Berezikov E, de BE, Horvitz HR, Kauppinen S, Plasterk RH: MicroRNA expression in zebrafish embryonic development. Science 2005, 309:310-311.

12. Fish JE, Santoro MM, Morton SU, Yu S, Yeh RF, Wythe JD, Ivey KN, Bruneau BG, Stainier DY, Srivastava D: miR-126 regulates angiogenic signaling and vascular integrity. Dev Cell 2008, 15:272-284.

13. Kuehbacher A, Urbich C, Zeiher AM, Dimmeler S: Role of Dicer and Drosha for endothelial microRNA expression and angiogenesis. Circ Res 2007, 101:59-68.

14. Wang S, Aurora AB, Johnson BA, Qi X, McAnally J, Hill JA, Richardson JA, Bassel-Duby R, Olson EN: The endothelial-specific microRNA miR-126 governs vascular integrity and angiogenesis. Dev Cell 2008, 15:261-271.

15. Nicoli S, Standley C, Walker P, Hurlstone A, Fogarty KE, Lawson ND: MicroRNA-mediated integration of haemodynamics and Vegf signalling during angiogenesis. Nature 2010, 464:1196-1200.

16. Guo C, Sah JF, Beard L, Willson JK, Markowitz SD, Guda K: The noncoding RNA, miR-126, suppresses the growth of neoplastic cells by targeting phosphatidylinositol 3-kinase signaling and is frequently lost in colon cancers. Genes Chromosomes Cancer 2008, 47:939-946.

17. Tavazoie SF, Alarcon C, Oskarsson T, Padua D, Wang Q, Bos PD, Gerald WL, Massague J: Endogenous human microRNAs that suppress breast cancer metastasis. Nature 2008, 451:147-152.

18. Crawford M, Brawner E, Batte K, Yu L, Hunter MG, Otterson GA, Nuovo G, Marsh CB, Nana-Sinkam SP: MicroRNA-126 inhibits invasion in non-small cell lung carcinoma cell lines. Biochem Biophys Res Commun 2008, 373:607-612.

19. Zhong M, Ma X, Sun C, Chen L: MicroRNAs reduce tumor growth and contribute to enhance cytotoxicity induced by gefitinib in non-small cell lung cancer. Chem Biol Interact 2010, 184:431-438.

20. Musiyenko A, Bitko V, Barik S: Ectopic expression of miR-126*, an intronic product of the vascular endothelial EGF-like 7 gene, regulates prostein translation and invasiveness of prostate cancer LNCaP cells. J Mol Med 2008, 86:313-322.

21. Liu B, Peng XC, Zheng XL, Wang J, Qin YW: MiR-126 restoration downregulate VEGF and inhibit the growth of lung cancer cell lines in vitro and in vivo. Lung Cancer 2009, 66:169-175.

22. Tong RT, Boucher Y, Kozin SV, Winkler F, Hicklin DJ, Jain RK: Vascular normalization by vascular endothelial growth factor receptor 2 blockade induces a pressure gradient across the vasculature and improves drug penetration in tumors. Cancer Res 2004, 64:3731-3736.

23. Jain RK: Normalization of tumor vasculature: an emerging concept in antiangiogenic therapy. Science 2005, 307:58-62.

24. Jain RK: Normalizing tumor vasculature with anti-angiogenic therapy: a new paradigm for combination therapy. Nat Med 2001, 7:987-989.

25. Wildiers H, Guetens $G$, De BG, Verbeken E, Landuyt B, Landuyt W, de Bruijn EA, van Oosterom AT: Effect of antivascular endothelial growth factor treatment on the intratumoral uptake of CPT-11. Br J Cancer 2003, 88:1979-1986

26. Nielsen BS, Jorgensen S, Fog JU, Sokilde R, Christensen IJ, Hansen U, Brunner N, Baker A, Moller S, Nielsen HJ: High levels of microRNA-21 in the stroma of colorectal cancers predict short disease-free survival in stage II colon cancer patients. Clin Exp Metastasis 2011, 28:27-38.

27. Jorgensen S, Baker A, Moller S, Nielsen BS: Robust one-day in situ hybridization protocol for detection of microRNAs in paraffin samples using LNA probes. Methods 2010, 52:375-381.

28. Vincenzi B, Santini D, Russo A, Gavasci M, Battistoni F, Dicuonzo G, Rocci L, Rosaria VM, Gebbia N, Tonini G: Circulating VEGF reduction, response and outcome in advanced colorectal cancer patients treated with cetuximab plus irinotecan. Pharmacogenomics 2007, 8:319-327.

29. Hansen TF, Garm Spindler KL, Andersen RF, Lindebjerg J, Brandslund I, Jakobsen A: The predictive value of genetic variations in the vascular endothelial growth factor A gene in metastatic colorectal cancer. Pharmacogenomics J 2010, 11:53-60.

30. Diaz R, Silva J, Garcia JM, Lorenzo Y, Garcia V, Pena C, Rodriguez R, Munoz C, Garcia F, Bonilla F, Dominguez G: Deregulated expression of miR-106a predicts survival in human colon cancer patients. Genes Chromosomes Cancer 2008, 47:794-802.

31. Schepeler T, Reinert JT, Ostenfeld MS, Christensen LL, Silahtaroglu AN, Dyrskjot L, Wiuf C, Sorensen FJ, Kruhoffer M, Laurberg S, Kauppinen S, Orntoft TF, Andersen CL: Diagnostic and prognostic microRNAs in stage II colon cancer. Cancer Res 2008, 68:6416-6424.

\section{Pre-publication history}

The pre-publication history for this paper can be accessed here: http://www.biomedcentral.com/1471-2407/12/83/prepub

doi:10.1186/1471-2407-12-83

Cite this article as: Hansen et al:: The predictive value of microRNA-126 in relation to first line treatment with capecitabine and oxaliplatin in patients with metastatic colorectal cancer. BMC Cancer 2012 12:83.

\section{Submit your next manuscript to BioMed Central and take full advantage of:}

- Convenient online submission

- Thorough peer review

- No space constraints or color figure charges

- Immediate publication on acceptance

- Inclusion in PubMed, CAS, Scopus and Google Scholar

- Research which is freely available for redistribution

Submit your manuscript at www.biomedcentral.com/submit
Biomed Central 